\title{
Smooth translation to maintain a healthy skin
}

This study ...
indicates
that protein
translation
control is
important for
epidermal
homeostasis

Different mRNA features can lead to ribosome stalling during translation elongation. Because the accumulation of stalled ribosomes on mRNAs is toxic, cells have evolved mechanisms to rescue and recycle stalled ribosomes by inducing their release and the degradation of the associated nascent polypeptides. Now, Watt and colleagues show that defective ribosome recycling impairs stem cell function in the skin, thereby compromising epidermal homeostasis.

The authors studied the effects of the loss of the ribosome rescue factor Pelota (Pelo; Dom34 in yeast) in mice, which is expressed in the skin dermis and epidermis. Dermalspecific deletion of Pelo resulted in mice with a normal lifespan and no detectable dermal abnormalities. However, embryonic deletion of Pelo in epidermal (keratin 14-expressing) cells, including stem cell populations, led to several epidermal defects.

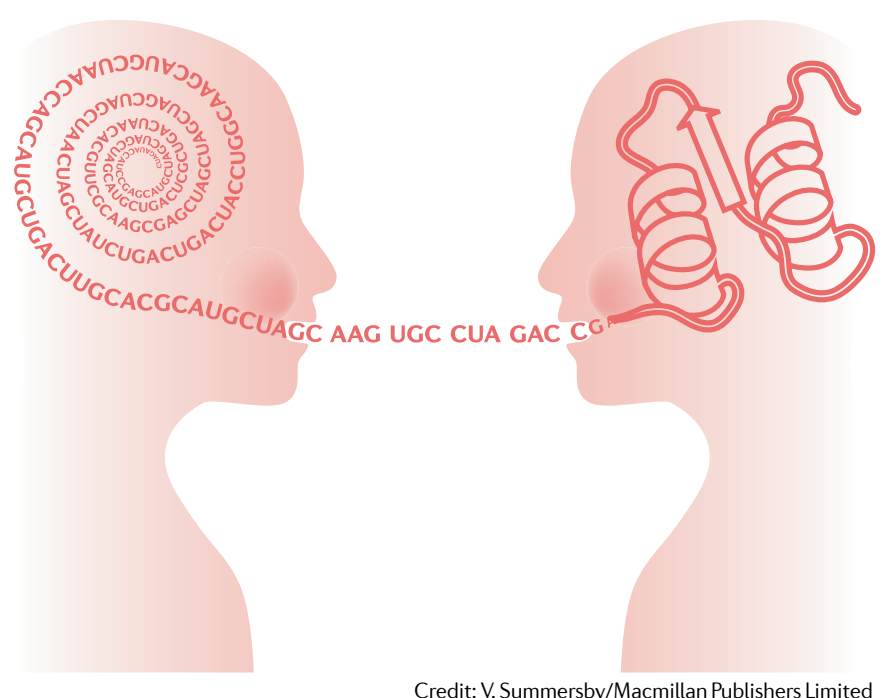

The mice had scaly skin, epidermal thickening due to overproliferation and accumulation of differentiated cells, increased transepidermal water loss (indicative of a defect in the barrier function of the skin) and delayed wound healing. Moreover, some hair follicle stem cell types were lost, leading to degeneration of hair follicles and sebaceous glands. Similar epidermal defects and skin lesions could be produced postnatally by inducing loss of Pelo in the epidermis of adult mice. Moreover, increased proliferation of basal layer cells was seen in human epidermis reconstituted on decellularised dermis using epidermal keratinocytes lacking Pelo.

Conditional knockout of Pelo in different mouse epidermal stem cell populations revealed that Pelo is required specifically in Lrig1-expressing cells to maintain epidermal homeostasis, as deletion of Pelo in Lrig $1^{+}$cells caused all the previously observed skin defects, whereas its deletion in Lgr5- or Lgr6-expressing cells had no effects or mild effects on skin.

Next, the authors investigated the consequences of Pelo knockout at the cellular level. They performed ribosome profiling (deep sequencing of ribosome-protected RNA fragments (RPFs)) on keratinocytes from adult mice lacking Pelo, which indicates to what extent and where ribosomes abnormally stall on mRNAs. The majority of RPFs were 28-34 nucleotides long, but there was an enrichment in 20-21 nucleotide RPFs ( 4-5\% of total RPFs compared to $<1 \%$ in control cells), which the authors suggested to be linked to quality control mechanisms previously characterised in yeast, which are initiated in response to tRNA starvation in rapidly dividing cells.

Surprisingly, epidermal loss of Pelo caused an increase in global translation efficiency in interfollicular epidermal cells, which was confirmed by the quantification of global protein synthesis. Gene expression profiling revealed an upregulation of genes involved in RNA metabolism and protein synthesis, among others, as well as upregulation of the mTOR pathway, which is known to promote global translation. Moreover, when Pelo was deleted, protein synthesis was increased in Lrig $1^{+}$cells relative to $\operatorname{Lgr}^{+}$and Lgr6 ${ }^{+}$cells, suggesting that the phenotype caused by the loss of Pelo primarily reflects increased translation rather than changes in the expression of specific genes. This finding was supported by the observation that inhibition of mTOR1 signalling largely rescued the defective epidermal phenotype caused by Pelo deletion.

This study establishes a link between Pelo inactivation (and thus ribosome stalling) and the compensatory activation of translation by mTOR signalling. Moreover, it indicates that protein translation control is important for epidermal homeostasis, adding to the growing body of evidence of translation being a key regulatory process of stem cell and tissue function.

Kim Baumann

ORIGINAL ARTICLE Liakath-Ali, K. et al. An evolutionary conserved ribosome-rescue pathway maintains epidermal homeostasis. Nature 556 , 376-380 (2018) 